\title{
SOME APPLICATIONS OF DEGENERATE POLY-BERNOULLI NUMBERS AND POLYNOMIALS
}

\author{
DAE SAN KIM AND TAEKYUN KIM
}

\begin{abstract}
In this paper, we consider degenerate poly-Bernoulli numbers and polynomials associated with polylogarithmic function and $p$-adic invariant integral on $\mathbb{Z}_{p}$. By using umbral calculus, we derive some identities of those numbers and polynomials.
\end{abstract}

\section{INTRODUCTION}

Let $p$ be a fixed prime number. Throughout this paper, $\mathbb{Z}_{p}, \mathbb{Q}_{p}$ and $\mathbb{C}_{p}$ will denote the ring of $p$-adic integers, the field of $p$-adic rational numbers and the completion of the algebraic closure of $\mathbb{Q}_{p}$. The $p$-adic norm is normalized as $|p|_{p}=\frac{1}{p}$. For $k \in \mathbb{Z}$, the polylogarithmic function $\operatorname{Li}_{k}(x)$ is defined by $\operatorname{Li}_{k}(x)=\sum_{n=1}^{\infty} \frac{x^{n}}{n^{k}}$. For $k=1$, we have $\operatorname{Li}_{1}(x)=-\log (1-x)$.

In [4], L. Carlitz considered the degenerate Bernoulli polynomials which are given by the generating function

$$
\frac{t}{(1+\lambda t)^{\frac{1}{\lambda}}-1}(1+\lambda t)^{\frac{x}{\lambda}}=\sum_{n=0}^{\infty} \beta_{n, \lambda}(x) \frac{t^{n}}{n !} .
$$

Note that $\lim _{\lambda \rightarrow 0} \beta_{n, \lambda}(x)=B_{n}(x)$, where $B_{n}(x)$ are the ordinary Bernoulli polynomials. When $x=0, \beta_{n, \lambda}=\beta_{n, \lambda}(0)$ are called the degenerate Bernoulli numbers.

It is known that the poly-Bernoulli polynomials are defined by the generating function

$$
\frac{\operatorname{Li}_{k}\left(1-e^{-t}\right)}{e^{t}-1} e^{x t}=\sum_{n=0}^{\infty} B_{n}^{(k)}(x) \frac{t^{n}}{n !}, \quad(\text { see }[\underline{8}]) .
$$

When $x=0, B_{n}^{(k)}=B_{n}^{(k)}(0)$ are called the poly-Bernoulli numbers.

Let $U D\left(\mathbb{Z}_{p}\right)$ be the space of uniformly differentiable functions on $\mathbb{Z}_{p}$. For $f \in$ $U D\left(\mathbb{Z}_{p}\right)$, the $p$-adic invariant integral on $\mathbb{Z}_{p}$ is defined by

$$
\begin{aligned}
\int_{\mathbb{Z}_{p}} f(x) d \mu_{0}(x) & =\lim _{N \rightarrow \infty} \sum_{x=0}^{p^{N}-1} f(x) \mu_{0}\left(x+p^{N} \mathbb{Z}_{p}\right) \\
& =\lim _{N \rightarrow \infty} \frac{1}{p^{N}} \sum_{x=0}^{p^{N}-1} f(x), \quad \text { (see [13]). }
\end{aligned}
$$

2010 Mathematics Subject Classification. 05A40, 11B83, 11S80.

Key words and phrases. Degenerate poly-Bernoulli polynomial, $p$-adic invariant integral, Umbral calculus. 
From (1.3), we have

$$
\int_{\mathbb{Z}_{p}} f(x+1) d \mu_{0}(x)-\int_{\mathbb{Z}_{p}} f(x) d \mu_{0}(x)=f^{\prime}(0),
$$

where $f^{\prime}(0)=\left.\frac{d f(x)}{d x}\right|_{x=0}($ see $[1-17])$.

By (1.4), we get

$$
\begin{aligned}
\int_{\mathbb{Z}_{p}}(1+\lambda t)^{(x+y) / \lambda} d \mu_{0}(y) & =\frac{\log (1+\lambda t)^{\frac{1}{\lambda}}}{(1+\lambda t)^{\frac{1}{\lambda}}-1}(1+\lambda t)^{\frac{x}{\lambda}} \\
& =\frac{\log (1+\lambda t)}{\lambda t} \frac{t}{(1+\lambda t)^{\frac{1}{\lambda}}-1}(1+\lambda t)^{\frac{x}{\lambda}} \\
& =\sum_{n=0}^{\infty}\left(\sum_{l=0}^{n}\left(\begin{array}{l}
n \\
l
\end{array}\right) \lambda^{n-l} D_{n-l} \beta_{l, \lambda}(x)\right) \frac{t^{n}}{n !}
\end{aligned}
$$

where $D_{n}$ are the Daehee numbers of the first kind given by the generating function

$$
\frac{\log (1+t)}{t}=\sum_{n=0}^{\infty} D_{n} \frac{t^{n}}{n !}, \quad(\text { see }[9]) .
$$

Let $\mathcal{F}=\left\{f(t)=\sum_{k=0}^{\infty} a_{k} \frac{t^{k}}{k !} \mid a_{k} \in \mathbb{C}_{p}\right\}$ be the algebra of formal power series in a single variable $t$. Let $\mathbb{P}$ be the algebra of polynomials in a single vairable $x$ over $\mathbb{C}_{p}$. We denote the action of the linear functional $L \in \mathbb{P}^{*}$ on a polynomial $p(x)$ by $\langle L \mid p(x)\rangle$, which is linearly extended as $\left\langle c L+c^{\prime} L^{\prime} \mid p(x)\right\rangle=c\langle L \mid p(x)\rangle+$ $c^{\prime}\left\langle L^{\prime} \mid p(x)\right\rangle$, where $c, c^{\prime} \in \mathbb{C}_{p}$. We define a linear functional on $\mathbb{P}$ by setting

$$
\left\langle f(t) \mid x^{n}\right\rangle=a_{n}, \quad \text { for all } n \geq 0 \text { and } f(t) \in \mathcal{F} .
$$

By (1.7), we easily get

$$
\left\langle t^{k} \mid x^{n}\right\rangle=n ! \delta_{n, k}, \quad(n, k \geq 0),
$$

where $\delta_{n, k}$ is the Kronecker's symbol (see [15]).

For $f_{L}(t)=\sum_{k=0}^{\infty}\left\langle L \mid x^{k}\right\rangle \frac{t^{k}}{k !}$, we have $\left\langle f_{L}(t) \mid x^{n}\right\rangle=\left\langle L \mid x^{n}\right\rangle$. The map $L \mapsto$ $f_{L}(t)$ is vector space isomorphism from $\mathbb{P}^{*}$ onto $\mathcal{F}$. Henceforth $\mathcal{F}$ denotes both the algebra of formal power series in $t$ and the vector space of all linear functionals on $\mathbb{P}$, and so an element $f(t)$ of $\mathcal{F}$ is thought of as both a formal power series and a linear functional. We call $\mathcal{F}$ the umbral algebra. The umbral calculus is the study of umbral algebra.

The order $o(f(t))$ of the non-zero power series $f(t)$ is the smallest integer $k$ for which the coefficient of $t^{k}$ does not vanish (see [10,15]). If $o(f(t))=1$ (respectively, $o(f(t))=0)$, then $f(t)$ is called a delta (respectively, an invertible) series.

For $o(f(t))=1$ and $o(g(t))=0$, there exists a unique sequence $s_{n}(x)$ of polynomials such that $\left\langle g(t) f(t)^{k} \mid s_{n}(x)\right\rangle=n ! \delta_{n, k}(n, k \geq 0)$. The sequence $s_{n}(x)$ is called the Sheffer sequence for $(g(t), f(t))$, and we write $s_{n}(x) \sim(g(t), f(t))$ (see [15]).

For $f(t) \in \mathcal{F}$ and $p(x) \in \mathbb{P}$, by (1.8), we get

$$
\left\langle e^{y t} \mid p(x)\right\rangle=p(y), \quad\langle f(t) g(t) \mid p(x)\rangle=\langle g(t) \mid f(t) p(x)\rangle=\langle f(t) \mid g(t) p(x)\rangle
$$


and

$$
f(t)=\sum_{k=0}^{\infty}\left\langle f(t) \mid x^{k}\right\rangle \frac{t^{k}}{k !}, \quad p(x)=\sum_{k=0}^{\infty}\left\langle t^{k} \mid p(x)\right\rangle \frac{x^{k}}{k !}, \quad \text { (see [15]). }
$$

From (1.10), we note that

$$
p^{(k)}(0)=\left\langle t^{k} \mid p(x)\right\rangle=\left\langle 1 \mid p^{(k)}(x)\right\rangle, \quad(k \geq 0),
$$

where $p^{(k)}(0)$ denotes the $k$-th derivative of $p(x)$ with respect to $x$ at $x=0$.

By (1.11), we get

$$
t^{k} p(x)=p^{(k)}(x)=\frac{d^{k}}{d x^{k}} p(x), \quad(k \geq 0) .
$$

In [15], it is known that

$$
s_{n}(x) \sim(g(t), f(t)) \Longleftrightarrow \frac{1}{g(\bar{f}(t))} e^{x \bar{f}(t)}=\sum_{n=0}^{\infty} s_{n}(x) \frac{t^{n}}{n !}, \quad\left(x \in \mathbb{C}_{p}\right),
$$

where $\bar{f}(t)$ is the compositional inverse of $f(t)$ such that $f(\bar{f}(t))=\bar{f}(f(t))=t$.

From (1.12), we can easily derive the following equation:

$$
e^{y t} p(x)=p(x+y), \quad \text { where } p(x) \in \mathbb{P}=\mathbb{C}_{p}[x] .
$$

In this paper, we study degenerate poly-Bernoulli numbers and polynomials associated with polylogarithm function and $p$-adic invariant integral on $\mathbb{Z}_{p}$. Finally, we give some identities of those numbers and polynomials which are derived from umbral calculus.

\section{Some APplications of DEgenerate POLY-BeRnoulli NUmbers}

Now, we consider the degenerate poly-Bernoulli polynomials which are given by the generating function

$$
\frac{\operatorname{Li}_{k}\left(1-(1+\lambda t)^{-\frac{1}{\lambda}}\right)}{(1+\lambda t)^{\frac{1}{\lambda}}-1} e^{x t}=\sum_{n=0}^{\infty} \beta_{n, \lambda}^{(k)}(x) \frac{t^{n}}{n !}, \quad(k \in \mathbb{Z}) .
$$

From (1.13) and (2.1), we have

$$
\beta_{n, \lambda}^{(k)}(x) \sim\left(\frac{(1+\lambda t)^{\frac{1}{\lambda}}-1}{\operatorname{Li}_{k}\left(1-(1+\lambda t)^{-\frac{1}{\lambda}}\right)}, t\right),
$$

and

$$
\beta_{n, \lambda}^{(k)}(x)=\sum_{l=0}^{n}\left(\begin{array}{l}
n \\
l
\end{array}\right) \beta_{l, \lambda}^{(k)} x^{n-l},
$$

where $\beta_{l, \lambda}^{(k)}=\beta_{l, \lambda}^{(k)}(0)$ are called the degenerate poly-Bernoulli numbers.

Thus, by (2.3), we get

$$
\begin{aligned}
\int_{x}^{x+y} \beta_{n, \lambda}^{(k)}(u) d u & =\frac{1}{n+1}\left\{\beta_{n+1, \lambda}^{(k)}(x+y)-\beta_{n+1, \lambda}^{(k)}(x)\right\} \\
& =\frac{e^{y t}-1}{t} \beta_{n, \lambda}^{(k)}(x) .
\end{aligned}
$$


Let $f(t)$ be the linear functional such that

$$
\langle f(t) \mid p(x)\rangle=\int_{\mathbb{Z}_{p}} \frac{\left(e^{t}-1\right) \operatorname{Li}_{k}\left(1-(1+\lambda t)^{-\frac{1}{\lambda}}\right)}{t\left((1+\lambda t)^{\frac{1}{\lambda}}-1\right)} p(x) d \mu_{0}(x)
$$

for all polynomials $p(x)$. Then it can be determined as follows: for any $p(x) \in \mathbb{P}$,

$$
\left\langle\frac{t}{e^{t}-1} \mid p(x)\right\rangle=\int_{\mathbb{Z}_{p}} p(x) d \mu_{0}(x) .
$$

Replacing $p(x)$ by $\frac{e^{t}-1}{t} h(t) p(x)$, for $h(t) \in \mathcal{F}$, we get

$$
\langle h(t) \mid p(x)\rangle=\int_{\mathbb{Z}_{p}} \frac{e^{t}-1}{t} h(t) p(x) d \mu_{0}(x) .
$$

In particular, for $h(t)=1$, we obtain

$$
\int_{\mathbb{Z}_{p}} \frac{e^{t}-1}{t} p(x) d \mu_{0}(x)=p(0) .
$$

Therefore, by (2.5) and (2.6), we obtain the following theorem as a special case.

Theorem 1. For $p(x) \in \mathbb{P}$, we have

$$
\begin{aligned}
& \left\langle\frac{\operatorname{Li}_{k}\left(1-(1+\lambda t)^{-\frac{1}{\lambda}}\right)}{(1+\lambda t)^{\frac{1}{\lambda}}-1} \mid p(x)\right\rangle \\
= & \int_{\mathbb{Z}_{p}} \frac{\left(e^{t}-1\right) \operatorname{Li}_{k}\left(1-(1+\lambda t)^{-\frac{1}{\lambda}}\right)}{t\left((1+\lambda t)^{\frac{1}{\lambda}}-1\right)} p(x) d \mu_{0}(x),
\end{aligned}
$$

and

$$
\begin{aligned}
& \left\langle\frac{\left(e^{t}-1\right) \operatorname{Li}_{k}\left(1-(1+\lambda t)^{-\frac{1}{\lambda}}\right)}{t\left((1+\lambda t)^{\frac{1}{\lambda}}-1\right)} \int_{\mathbb{Z}_{p}} e^{y t} d \mu_{0}(y) \mid p(x)\right\rangle \\
= & \int_{\mathbb{Z}_{p}} \frac{\left(e^{t}-1\right) \operatorname{Li}_{k}\left(1-(1+\lambda t)^{-\frac{1}{\lambda}}\right)}{t\left((1+\lambda t)^{\frac{1}{\lambda}}-1\right)} p(x) d \mu_{0}(x) .
\end{aligned}
$$

In particular,

$$
\beta_{n, \lambda}^{(k)}=\left\langle\frac{\left(e^{t}-1\right) \operatorname{Li}_{k}\left(1-(1+\lambda t)^{-\frac{1}{\lambda}}\right)}{t\left((1+\lambda t)^{\frac{1}{\lambda}}-1\right)} \int_{\mathbb{Z}_{p}} e^{y t} d \mu_{0}(y) \mid x^{n}\right\rangle, \quad(n \geq 0) .
$$

Note that

$$
\begin{aligned}
& \left\langle\int_{\mathbb{Z}_{p}} e^{y t} d \mu_{0}(y) \mid \frac{e^{t}-1}{t} \beta_{n, \lambda}^{(k)}(x)\right\rangle \\
= & \frac{1}{n+1}\left\langle\frac{t}{e^{t}-1} \mid \beta_{n+1, \lambda}^{(k)}(x+1)-\beta_{n+1, \lambda}^{(k)}(x)\right\rangle
\end{aligned}
$$




$$
=\frac{1}{n+1} \sum_{l=0}^{n+1}\left(\begin{array}{c}
n+1 \\
l
\end{array}\right) B_{l}\left(\beta_{n+1-l, \lambda}^{(k)}(1)-\beta_{n+1-l, \lambda}^{(k)}\right)=\beta_{n, \lambda}^{(k)} .
$$

It is easy to show that

$$
\begin{aligned}
& \frac{\left(e^{t}-1\right) \operatorname{Li}_{k}\left(1-(1+\lambda t)^{-\frac{1}{\lambda}}\right)}{t\left((1+\lambda t)^{\frac{1}{\lambda}}-1\right)} \sum_{n=0}^{\infty} \int_{\mathbb{Z}_{p}}(x+y)^{n} d \mu_{0}(y) \frac{t^{n}}{n !} \\
& =\frac{\left(e^{t}-1\right) \operatorname{Li}_{k}\left(1-(1+\lambda t)^{-\frac{1}{\lambda}}\right)}{t\left((1+\lambda t)^{\frac{1}{\lambda}}-1\right)} \times \frac{t}{e^{t}-1} e^{x t} \\
& =\sum_{n=0}^{\infty} \beta_{n, \lambda}^{(k)}(x) \frac{t^{n}}{n !} .
\end{aligned}
$$

Thus, by (2.7), we get

$$
\begin{aligned}
\beta_{n, \lambda}^{(k)}(x) & =\frac{\left(e^{t}-1\right) \operatorname{Li}_{k}\left(1-(1+\lambda t)^{-\frac{1}{\lambda}}\right)}{t\left((1+\lambda t)^{\frac{1}{\lambda}}-1\right)} \int_{\mathbb{Z}_{p}}(x+y)^{n} d \mu_{0}(y) \\
& =\frac{\operatorname{Li}_{k}\left(1-(1+\lambda t)^{-\frac{1}{\lambda}}\right)}{(1+\lambda t)^{\frac{1}{\lambda}}-1} x^{n}
\end{aligned}
$$

Therefore, by (2.8), we obtain the following theorem.

Theorem 2. For $p(x) \in \mathbb{P}$, we have

$$
\begin{aligned}
& \frac{\left(e^{t}-1\right) \operatorname{Li}_{k}\left(1-(1+\lambda t)^{-\frac{1}{\lambda}}\right)}{t\left((1+\lambda t)^{\frac{1}{\lambda}}-1\right)} \int_{\mathbb{Z}_{p}} p(x+y) d \mu_{0}(y) \\
= & \frac{\left(e^{t}-1\right) \operatorname{Li}_{k}\left(1-(1+\lambda t)^{-\frac{1}{\lambda}}\right)}{t\left((1+\lambda t)^{\frac{1}{\lambda}}-1\right)} \int_{\mathbb{Z}_{p}} e^{y t} p(x) d \mu_{0}(y) \\
= & \frac{\operatorname{Li}_{k}\left(1-(1+\lambda t)^{-\frac{1}{\lambda}}\right)}{(1+\lambda t)^{\frac{1}{\lambda}}-1} p(x) .
\end{aligned}
$$

For $r \in \mathbb{N}$, let us consider the higher-order degenerate poly-Bernoulli polynomials as follows:

$$
\begin{aligned}
& \left(\frac{\left(e^{t}-1\right) \operatorname{Li}_{k}\left(1-(1+\lambda t)^{-\frac{1}{\lambda}}\right)}{t\left((1+\lambda t)^{\frac{1}{\lambda}}-1\right)}\right)^{r} \int_{\mathbb{Z}_{p}} \cdots \int_{\mathbb{Z}_{p}} e^{\left(x_{1}+\cdots+x_{r}+x\right) t} d \mu_{0}\left(x_{1}\right) \cdots d \mu_{0}\left(x_{r}\right) \\
= & \left(\frac{\operatorname{Li}_{k}\left(1-(1+\lambda t)^{-\frac{1}{\lambda}}\right)}{(1+\lambda t)^{\frac{1}{\lambda}}-1}\right)^{r} e^{x t}=\sum_{n=0}^{\infty} \beta_{n, \lambda}^{(k, r)}(x) \frac{t^{n}}{n !} .
\end{aligned}
$$


Thus, we obtain

$$
\begin{aligned}
\beta_{n, \lambda}^{(k, r)}(x)= & \left(\frac{\operatorname{Li}_{k}\left(1-(1+\lambda t)^{-\frac{1}{\lambda}}\right)}{(1+\lambda t)^{\frac{1}{\lambda}}-1}\right)^{r} x^{n} \\
= & \left(\frac{\left(e^{t}-1\right) \operatorname{Li}_{k}\left(1-(1+\lambda t)^{-\frac{1}{\lambda}}\right)}{t\left((1+\lambda t)^{\frac{1}{\lambda}}-1\right)}\right)^{r} \\
& \times \int_{\mathbb{Z}_{p}} \cdots \int_{\mathbb{Z}_{p}}\left(x_{1}+\cdots+x_{r}+x\right)^{n} d \mu_{0}\left(x_{1}\right) \cdots d \mu_{0}\left(x_{r}\right),
\end{aligned}
$$

where $n \geq 0$.

Here, for $x=0, \beta_{n, \lambda}^{(k, r)}=\beta_{n, \lambda}^{(k, r)}(0)$ are called the degenerate poly-Bernoulli numbers of order $r$. From (2.9), we note that

$$
\beta_{n, \lambda}^{(k)}(x) \sim\left(\left(\frac{(1+\lambda t)^{\frac{1}{\lambda}}-1}{\operatorname{Li}_{k}\left(1-(1+\lambda t)^{-\frac{1}{\lambda}}\right)}\right)^{r}, t\right) .
$$

Therefore, by (2.10), we obtain the following theorem.

Theorem 3. For $p(x) \in \mathbb{P}$ and $r \in \mathbb{N}$, we have

$$
\begin{aligned}
& \left(\frac{\left(e^{t}-1\right) \operatorname{Li}_{k}\left(1-(1+\lambda t)^{-\frac{1}{\lambda}}\right)}{t\left((1+\lambda t)^{\frac{1}{\lambda}}-1\right)}\right) \int_{\mathbb{Z}_{p}} \cdots \int_{\mathbb{Z}_{p}} p\left(x_{1}+\cdots+x_{r}+x\right) d \mu_{0}\left(x_{1}\right) \cdots d \mu_{0}\left(x_{r}\right) \\
= & \left(\frac{\left(e^{t}-1\right) \operatorname{Li}_{k}\left(1-(1+\lambda t)^{-\frac{1}{\lambda}}\right)}{t\left((1+\lambda t)^{\frac{1}{\lambda}}-1\right)}\right)^{r} \int_{\mathbb{Z}_{p}} \cdots \int_{\mathbb{Z}_{p}} e^{\left(x_{1}+\cdots+x_{r}\right) t} p(x) d \mu_{0}\left(x_{1}\right) \cdots d \mu_{0}\left(x_{r}\right) \\
= & \left(\frac{\operatorname{Li}_{k}\left(1-(1+\lambda t)^{\frac{1}{\lambda}}\right)}{(1+\lambda t)^{\frac{1}{\lambda}}-1}\right)^{r} p(x) .
\end{aligned}
$$

Let us consider the linear functional $f_{r}(t)$ such that

$$
\begin{aligned}
& \left\langle f_{r}(t) \mid p(x)\right\rangle \\
= & \left.\int_{\mathbb{Z}_{p}} \cdots \int_{\mathbb{Z}_{p}}\left(\frac{\left(e^{t}-1\right) \operatorname{Li}_{k}\left(1-(1+\lambda t)^{-\frac{1}{\lambda}}\right)}{t\left((1+\lambda t)^{\frac{1}{\lambda}}-1\right)}\right)^{r} p(x)\right|_{x=x_{1}+\cdots+x_{r}} d \mu_{0}\left(x_{1}\right) \cdots d \mu_{0}\left(x_{r}\right)
\end{aligned}
$$

for all polynomials $p(x)$. Then it can be determined in the following way: for $p(x) \in \mathbb{P}$,

$$
\left\langle\left(\frac{t}{e^{t}-1}\right)^{r} \mid p(x)\right\rangle=\left.\int_{\mathbb{Z}_{p}} \cdots \int_{\mathbb{Z}_{p}} p(x)\right|_{x=x_{1}+\cdots+x_{r}} d \mu_{0}\left(x_{1}\right) \cdots d \mu_{0}\left(x_{r}\right) .
$$


Replacing $p(x)$ by $\left(\frac{e^{t}-1}{t} h(t)\right)^{r} p(x)$, for $h(t) \in \mathcal{F}$, we have

$$
\left\langle h(t)^{r} \mid p(x)\right\rangle=\left.\int_{\mathbb{Z}_{p}} \cdots \int_{\mathbb{Z}_{p}}\left(\frac{e^{t}-1}{t} h(t)\right)^{r} p(x)\right|_{x=x_{1}+\cdots+x_{r}} d \mu_{0}\left(x_{1}\right) \cdots d \mu_{0}\left(x_{r}\right) .
$$

In particular, for $h(t)=1$, we get

$$
\left.\int_{\mathbb{Z}_{p}} \cdots \int_{\mathbb{Z}_{p}}\left(\frac{e^{t}-1}{t}\right)^{r} p(x)\right|_{x=x_{1}+\cdots+x_{r}} d \mu_{0}\left(x_{1}\right) \cdots d \mu_{0}\left(x_{r}\right)=p(0) .
$$

Therefore, by (2.13) and (2.14), we obtain the following theorem.

Theorem 4. For $p(x) \in \mathbb{P}$, we have

$$
\begin{aligned}
& \left\langle\left(\frac{\operatorname{Li}_{k}\left(1-(1+\lambda t)^{-\frac{1}{\lambda}}\right)}{(1+\lambda t)^{\frac{1}{\lambda}}-1}\right)^{r} \mid p(x)\right\rangle \\
= & \left.\int_{\mathbb{Z}_{p}} \cdots \int_{\mathbb{Z}_{p}}\left(\frac{\left(e^{t}-1\right) \operatorname{Li}_{k}\left(1-(1+\lambda t)^{-\frac{1}{\lambda}}\right)}{t\left((1+\lambda t)^{\frac{1}{\lambda}}-1\right)}\right)^{r} p(x)\right|_{x=x_{1}+\cdots+x_{r}} d \mu_{0}\left(x_{1}\right) \cdots d \mu_{0}\left(x_{r}\right), \\
\text { and } & \left\langle\left(\frac{\left(e^{t}-1\right) \operatorname{Li}_{k}\left(1-(1+\lambda t)^{-\frac{1}{\lambda}}\right)}{t\left((1+\lambda t)^{\frac{1}{\lambda}}-1\right)^{r} \int_{\mathbb{Z}_{p}} \cdots \int_{\mathbb{Z}_{p}} e^{\left(x_{1}+\cdots+x_{r}\right) t} d \mu_{0}\left(x_{1}\right) \cdots d \mu_{0}\left(x_{r}\right)|p(x)\rangle}|\rangle\right.\right. \\
& \left\langle\left.\int_{\mathbb{Z}_{p}} \cdots \int_{\mathbb{Z}_{p}}\left(\frac{\left(e^{t}-1\right) \operatorname{Li}_{k}\left(1-(1+\lambda t)^{-\frac{1}{\lambda}}\right)}{t\left((1+\lambda t)^{\frac{1}{\lambda}}-1\right)}\right)^{r} p(x)\right|_{x=x_{1}+\cdots+x_{r}} d \mu_{0}\left(x_{1}\right) \cdots d \mu_{0}\left(x_{r}\right) .\right.
\end{aligned}
$$

In particular,

$$
\beta_{n, \lambda}^{(k, r)}=\left\langle\left(\frac{\left(e^{t}-1\right) \operatorname{Li}_{k}\left(1-(1+\lambda t)^{-\frac{1}{\lambda}}\right)}{t\left((1+\lambda t)^{\frac{1}{\lambda}}-1\right)}\right)^{r} \int_{\mathbb{Z}_{p}} \cdots \int_{\mathbb{Z}_{p}} e^{\left(x_{1}+\cdots+x_{r}\right) t} d \mu_{0}\left(x_{1}\right) \cdots d \mu_{0}\left(x_{r}\right) \mid x^{n}\right\rangle .
$$

Remark. It is not difficult to show that

$$
\begin{aligned}
& \left\langle\left(\frac{\left(e^{t}-1\right) \operatorname{Li}_{k}\left(1-(1+\lambda t)^{-\frac{1}{\lambda}}\right)}{t\left((1+\lambda t)^{\frac{1}{\lambda}}-1\right)}\right)^{r} \int_{\mathbb{Z}_{p}} \ldots \int_{\mathbb{Z}_{p}} e^{\left(x_{1}+\cdots+x_{r}\right) t} d \mu_{0}\left(x_{1}\right) \cdots d \mu_{0}\left(x_{r}\right) \mid x^{n}\right\rangle \\
= & \sum_{n=n_{1}+\cdots+n_{r}}\left(\begin{array}{c}
n \\
n_{1}, \ldots, n_{r}
\end{array}\right)\left\langle\frac{\left(e^{t}-1\right) \operatorname{Li}_{k}\left(1-(1+\lambda t)^{-\frac{1}{\lambda}}\right)}{t\left((1+\lambda t)^{\frac{1}{\lambda}}-1\right)} \int_{\mathbb{Z}_{p}} e^{x_{n_{1}} t} d \mu_{0}\left(x_{1}\right) \mid x^{m_{1}}\right\rangle \times \cdots \\
& \times\left\langle\frac{\left(e^{t}-1\right) \operatorname{Li}_{k}\left(1-(1+\lambda t)^{-\frac{1}{\lambda}}\right)}{t\left((1+\lambda t)^{\frac{1}{\lambda}}-1\right)} \int_{\mathbb{Z}_{p}} e^{x_{n_{r}} t} d \mu_{0}\left(x_{n_{r}}\right) \mid x^{n_{r}}\right\rangle .
\end{aligned}
$$

Thus, we get

$$
\beta_{n, \lambda}^{(k, r)}=\sum_{n=n_{1}+\cdots+n_{r}}\left(\begin{array}{c}
n \\
n_{1}, \ldots, n_{r}
\end{array}\right) \beta_{n_{1}, \lambda}^{(k)} \cdots \beta_{n_{r}, \lambda}^{(k)}
$$




\section{REFERENCES}

1. S. Araci, M. Acikgoz, and A. Kilicman, Extended p-adic q-invariant integrals on $\mathbb{Z}_{p}$ associated with applications of umbral calculus, Adv. Difference Equ. (2013), 2013:96, 14. MR 3055847

2. S. Araci, M. Acikgoz, and E. Sen, On the Kim's p-adic q-deformed fermionic integral in the p-adic integer ring, J. Number Theory 133 (2013), no. 10, 33483361. MR 3071817

3. A. Bayad and T. Kim, Identities involving values of Bernstein, q-Bernoulli, and q-Euler polynomials, Russ. J. Math. Phys. 18 (2011), no. 2, 133-143. MR 2810987 (2012d:11039)

4. L. Carlitz, Degenerate Stirling, Bernoulli and Eulerian numbers, Utilitas Math. 15 (1979), 51-88. MR 531621 (80i:05014)

5. S. Gaboury, R. Tremblay, and B.-J. Fugère, Some explicit formulas for certain new classes of Bernoulli, Euler and Genocchi polynomials, Proc. Jangjeon Math. Soc. 17 (2014), no. 1, 115-123. MR 3184467

6. Y. He and W. Zhang, A convolution formula for Bernoulli polynomials, Ars Combin. 108 (2013), 97-104. MR 3060257

7. F. T. Howard Explicit formulas for degenerate Bernoulli numbers, Discrete Math. 162 (1996), 175-185. MR 1425786

8. D. Kim and T. Kim, A note on poly-Bernoulli and higher-order poly-Bernoulli polynomials, Russ. J. Math. Phys. 22 (2015), no. 1, 26-33. MR 3318873

9. D. S. Kim and T. Kim, Daehee Polynomials with q-parameter, Adv. Studies. Theor. Phys. 8 (2014), no. 13, 561-569.

10. _ Higher-order Frobenius-Euler and poly-Bernoulli mixed-type polynomials, Adv. Difference Equ. (2013), 2013:251, 13. MR 3108262

11. T. Kim, q-Volkenborn integration, Russ. J. Math. Phys. 9 (2002), no. 3, 288299. MR 1965383 (2004f:11138)

12. (1994), no. 1, 73-86. MR 1269069 (95c:11140)

13. Symmetry p-adic invariant integral on $\mathbb{Z}_{p}$ for Bernoulli and Euler polynomials, J. Difference Equ. Appl. 14 (2008), no. 12, 1267-1277. MR 2462529 (2009i:11023)

14. H. Ozden, p-adic distribution of the unification of the Bernoulli, Euler and Genocchi polynomials, Appl. Math. Comput. 218 (2011), no. 3, 970-973. MR 2831340

15. S. Roman, The umbral calculus, Pure and Applied Mathematics, vol. 111, Academic Press, Inc. [Harcourt Brace Jovanovich, Publishers], New York, 1984. MR 741185 (87c:05015)

16. O. Schnetz, Graphical functions and single-valued multiple polylogarithms, Commun. Number Theory Phys.8 (2014), no. 4, 589-675. MR 3318386

17. C. F. Woodcock, An invariant $p$-adic integral on $Z_{p}$, J. London Math. Soc. (2) 8 (1974), 731-734. MR 0352057 (50 \#4545)

Department of Mathematics, Sogang University, Seoul 121-742, Republic of Korea

E-mail address: dskim@sogang.ac.kr

Department of Mathematics, Kwangwoon University, Seoul 139-701, Republic of KoREA

E-mail address: tkkim@kw.ac.kr 